\title{
Clinicopathological Characteristics of Testicular Tumors
}

\author{
Sumat $\mathrm{K}^{1}$, Rumana $\mathrm{MH}^{2 *}$ and Parveen Shah ${ }^{3}$ \\ ${ }^{1}$ Department of pathology, SKIMS, Soura, India \\ ${ }^{2}$ Department of pathology, Sheri Kashmir Institute of Medical Sciences, Soura, India \\ ${ }^{3}$ Professor and Head of department, Department of Pathology, SKIMS, Soura, India
}

*Corresponding author: Makhdoomi RH, professor of pathology, Fellowship

neuropathology, Shere -Kashmir Institute of Medical Sciences, Soura, India, Email: rumanamak@gmail.com

\section{Review Article \\ Volume 1 Issue 1}

Received Date: June 29, 2017

Published Date: July 26, 2017

DOI: $10.23880 /$ cprj-16000106

\section{Abstract}

Testicular tumors constitute one of the most common tumors of men. Of all the testicular tumors, Germ cell tumors (GCT's) comprise 95\%. Others include sex-cord stromal tumors, mixed germ cell sex-cord stromal tumors, hematopoietic tumors, miscellaneous tumors and secondary tumors. A number of pre-existing medical conditions have been associated with the development of TGCT (testicular germ cell tumour). Most patients of testicular neoplasms present with progressive painless enlargement of testis, history of trauma and hydrocele. Testicular ultrasonography is used to determine the location of a palpable mass when testicular cancer is suspected. Generally, palpable extra-testicular lesions are benign. On the other hand, intratesticular masses, especially if they are palpable, are likely to be malignant and must be surgically explored. The vast majorities of testicular tumors are of germ cell origin and, like the totipotent germ cells from which they arise, may differentiate along several pathways

Keywords: Testicular Tumor; GCNIS (germ cell neoplasia in situ) Seminoma; Non Seminomatous Germ Cell Tumor; Cryptorchidism; Serum Markers

\section{Introduction}

Testicular neoplasms span an amazing gamut of anatomic types. They constitute one of the most common tumors of men. The incidence increases shortly after the onset of puberty and reaches maximum in men in late twenties and thirties (15-34 years). It decreases in men in their sixties and older. A distinct peak in incidence of testicular tumors occurs in infants. Testicular tumors comprise approximately $1 \%$ of all cancers in men, but only about $0.1 \%$ of cancer deaths in males because the majority of these tumors are curable [1-3]. However for unclear reasons, the incidence of testicular tumors has increased progressively in twentieth century.
Of all the testicular tumors, Germ cell tumors (GCT's) comprise $95 \%$ others include sex-cord stromal tumors, mixed germ cell sex-cord stromal tumors, hematopoietic tumors, miscellaneous tumors and secondary tumors. Histologically, germ cell tumors are divided into two major groups i.e. Seminomas and Non-seminomatous germ cell tumors (NSGCT). In WHO classification 2016 germ cell tumours are broadly classified into two categories germ cell tumor derived for germ cell neoplasia in situ and germ cell tumor unrelated to germ cell neoplasia in situ. The former category is further divided into four categories including Non invasive germ cell neoplasia, tumors of single histological type, non seminomatous germ cell tumor of more than one 
histological type, germ cell tumors of unknown type. Seminoma is commonest tumour of single histological type and accounts for approximately $50 \%$ of the testicular tumors, differentiate along the gonadal cell lines, and resemble primordial germ cells and gonocytes. NSGCT (Non seminomatous germ cell tumors) in turn, have retained pluripotency and include a number of different subgroups. These tumors display various stages of embryonic differentiation; ranging from highly undifferentiated cells resembling embryonic stem cells in the embryonal carcinomas, to the highly differentiated cells of somatic tissue in the teratomas. NSGCT's may also present as extra embryonic tissue, that is, as yolk sac tumors or as choriocarcinomas. NSGCT's comprise $40 \%$ of testicular cancers. The remaining $10 \%$ of testicular cancers are combined tumors containing both seminomatous and non-seminoma to us elements [1,4-6].

Extragonadal germ cell tumors account for 1-5\% of all germ cell tumors. The most widely accepted theory suggests that extragonadal germ cell tumors arise from primordial germ cells misplaced during their migration to gonads. Klinefelter syndrome is the only known predisposing factor. The clinical course is variable, with a potential for aggressive behavior and widespread metastases. Extra-gonadal germ cell tumors are considered as metastases from occult or "burned out" gonadal cancer until proved otherwise. If a primary testicular tumor is detected, the extra-gonadal mass is always considered metastatic. They affect men during third and fourth decade of life.

Testicular pathology is a nosologically complex subject because of the spectrum of histologic subtypes and variable clinical behavior, particular among GCTs. This diversity is best reflected in various systems offered to classify these tumors. Currently the most comprehensive and widely accepted system of classification is the one proposed by the World Health Organization (WHO) [2] in 2004 and the recent revised WHO classification of 2016 [1].

Biologic behavior depends upon the types of tumors present, as well as clinical features, including the age of the patient and primary site (testicular versus retroperitoneal), since histologically identical tumors can behave differently depending upon these clinical parameters. Clinically, NSGCT's have a more aggressive behavior, are less susceptible to radiation, and manifest at an earlier age. Moreover, mature teratoma, in contrast to other tumors, are less sensitive to platinum-based chemotherapy. These differences indicate etiological heterogeneity between the two groups.
High inguinal orchiectomy is the standard initial treatment for suspected testicular carcinoma. This allows accurate staging and histological diagnosis of the tumor, while ensuring the best local control and minimizing treatment morbidity. However, the tumor is remarkably sensitive to chemotherapy and is a model for a highly curable, advanced cancer. Appropriate integration of surgery, chemotherapy and radiation therapy in treatment has lead to high cure rates. The introduction of Cisplastin-based chemotherapy has revolutionized the management of testicular tumors, with considerable improvement in response rates and excellent outcome reported even in presence of metastatic disease [7].

\section{Etiology \& Risk Factors}

A number of pre-existing medical conditions have been associated with the development of TGCT (testicular germ cell tumour). These conditions include prior diagnosis of TGCT in the contralateral testicle, cryptorchidism, impaired spermatogenesis, inguinal hernia, hydrocele, disorders of sex development, prior testicular biopsy, atopy and testicular atrophy [8].

Cryptorchidism, or undescended testis, is the antecedent medical condition most closely associated with TGCT. A meta-analysis has estimated the relative risk of TGCT among men with prior cryptorchidism to be 4.8 (95\%CI $=4.0-5.7$ ) [9]. Nevertheless, only $10 \%$ of TGCTs develop in men with prior cryptorchidism. High birth weight has also been associated with TGCT in at least one study [10], the majority of studies have not supported a relationship [8].

In general, studies have found that men with TGCT have higher follicle stimulating hormone (FSH) levels and somewhat lower testosterone levels than do control men. Studies in cryptorchid men have reported a similar hormonal milieu [11]. The associations of reduced bodymuscle mass and reduced frequency of baldness among men with TGCT have also suggested that testosterone levels in TGCT patients may be in the lower end of the spectrum [12].

Studies have found an increased risk of TGCT among firefighters, metal workers, leather workers, aircraft technicians, agricultural workers, unionized carpenters, paper mill maintenance employees and writers. In general, white collar workers have been found to be at higher risk of TGCT than blue collar workers. Thus occupational associations could be confounded by socioeconomic status [13]. 
An infectious etiology of testicular cancer has been suggested. Recent support for a viral etiology has been the reports of increased risk of TGCT among men infected with human immunodeficiency virus (HIV) [14].

The majority of risk factor analyses stratified by histologic group appear to support a shared etiopathogenesis [8]. Several studies have reported that cryptorchidism, low birth weight and low birth order are factors predominantly associated with an increased risk of seminoma [8].

In comparison with the general population, the risk of testicular cancer has been reported to be eight-fold higher in brothers and four-fold higher in sons of affected men [15]. Despite the evidence that testicular cancer has a genetic component, it is likely that the risk is largely mediated by environmental exposures.

The onset of testicular cancer is insidious, and there are few symptoms. The diagnosis of testicular cancer is based on histopathologic evaluation of the testis after orchiectomy or rarely, biopsy of a retroperitoneal mass or subclavian lymph node. Such extraprimary/extragonadal disease constitutes less than $5 \%$ of cases.

Other than genital examinations during routine physical examination, no formal screening algorithm exists for testicular cancer. Routine ultrasound examinations and serologic testing are not cost-effective.

\section{Clinical Features}

Presenting Complaints: Patients of testicular tumor present with different complaints. Most patients present with progressive painless enlargement of testis, history of trauma and hydrocele. Tan G.H. et al., reported testicular swelling in $100 \%$ of cases while Shirish C. et al., reported scrotal swelling in $96 \%$ of cases $[16,17]$. Testicular pain was observed in 14-24.8\% of patients with testicular tumors as reported by various authors. Secondary hydrocele is observed in testicular tumors due to reactionary fluid accumulation between tunica vaginalis and tunica albugenia. In a study done by Mahalik SK, et al. $50 \%$ of patients with testicular tumors had associated hydrocele [18-20].
Age of Patients: There is good correlation between age and incidence of different types of testicular tumors testicular tumors. The peak is 41.9 years for classic seminoma, 65 years for spermatocytic seminoma, 30.4 years for the different types of NSGCT, and 35.1 years for the combination of seminoma and NSGCT. In prepubertal children, seminomas and combined tumors are rare. Only NSGCT occur with some frequency, notably of the yolk sac tumor and the mature teratoma types [19]. Fischer CG, et al. [20] from Germany reported highest age related incidence in two age groups $25-29$ years and 30-35 years. Albrecht W. et al from Veinna Austria (2004) noted testicular tumors more frequently in 15-34 years of age [21]. Salako AA, et al. in a retrospective study from Nigeria (2010) reported maximum number of cases in 2039 years of age [22].

\section{Laterality and Un-Descended Testes}

A predilection for the right side is well established although unexplained feature of testicular cancer. Right side preponderance has been reported worldwide in various series by different authors [17]. In various other studies, the incidence of bilateral testicular tumors varies from 0.5-7\%. Collins and Pugh (1964) recorded a frequency of $2.3 \%$. Some authors recorded a frequency of $1 \%$. Cryptorchidism (undescended testis) is consistently associated with an increased risk of testicular germ cell tumor. The incidence is about 3-5 fold increased in men with a history of cryptorchidism. The most common type of tumor in cryptorchid testis is seminoma believed to be derived from pleuripotent prenatal germ cells [23].

\section{Radiological Characteristics}

Ultrasonography is the standard imaging technique used to identify testicular carcinoma. It has a high sensitivity, but it must be combined with physical examination to achieve the best specificity. More than $95 \%$ of testicular parenchymal abnormalities are identifiable on routine sonograms, but several other lesions commonly mimic testicular cancer (Figure 1A). Ultrasonography is more specific in presence of a palpable mass. Non palpable intratesticular lesions larger than $1 \mathrm{~cm}$ are unlikely to be malignant $[24,25]$. The falsenegative rate for ultrasonographic identification of testicular lesions is extremely low. 


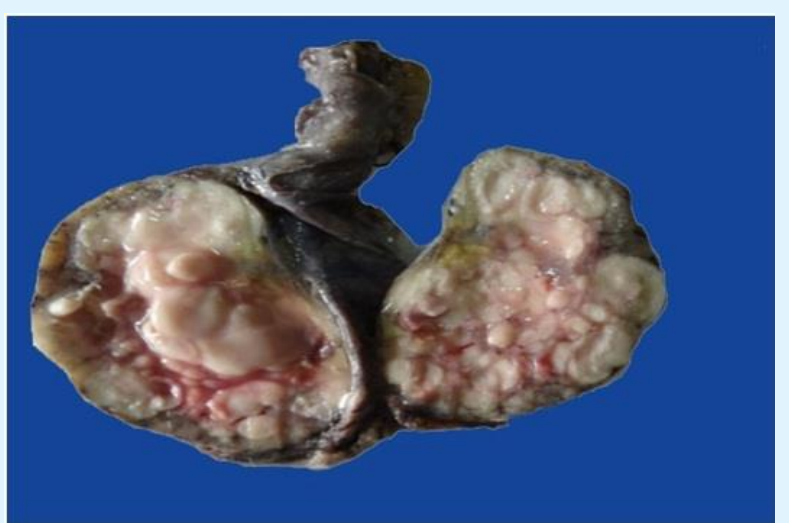

Figure 1A: Gross specimen of testis with Seminoma. Cut section showing well encapsulated growth replacing whole testicular parenchyma. Areas of hemorrhage and necrosis are also seen.

Testicular swelling or pain warrants a scrotal ultrasonography. Ultrasound images of seminoma are more often hypoechoic, homogeneous, and lobulated than those of non-seminoma ( $p<0.001$ ). Images of nonseminoma are more often heterogeneous and cystic $(\mathrm{p}<$ 0.001). There is no difference in tumor size, multiplicity, presence of calcium or lesion margination. Testicular microlithiasis is more common in seminoma ( $<<0.02)$. These observations were based on a study of 107 patients of testicular cancer by McDonald MW, et al. in 2012 [26].

Testicular ultrasonography is used to determine the location of a palpable mass when testicular cancer is suspected. Generally, palpable extra-testicular lesions are benign. On the other hand, intratesticular masses, especially if they are palpable, are likely to be malignant and must be surgically explored. Therefore, ultrasonography is useful for localizing palpable abnormalities and to triage them for surgical repair when indicated [27].

Testicular cancers are hypoechoic relative to the surrounding parenchyma in about $95 \%$ of cases. Published findings suggest that seminomas are often more homogeneously hypoechoic and that nonseminomatous lesions are often more cystic, with interspersed areas of calcification [28].

\section{Clinical Pathology \& Research Journal}

\section{Computed Tomography}

CT scan imaging is routinely performed as part of the initial staging process, but it is not sensitive or specific enough to be useful in evaluating undiagnosed testicular masses. Chest, abdominal, and pelvic CT scan studies are indicated for the evaluation of retroperitoneal and mediastinal metastases. Both computed tomography (CT) scanning and ultrasonography have been used to search for metastatic retroperitoneal lymphadenopathy, but CT scanning is more commonly used.

\section{Magnetic Resonance Imaging}

Ultrasonographic findings may occasionally be inconclusive in the evaluation of scrotal masses. Magnetic Resonance imaging may provide additional information in these cases, often affecting patient management [29]. Increased signal intensity in the testicle is seen on T2weighted images of testicular malignancies, but it is not specific.

\section{Tumor Markers}

There are three relatively sensitive and specific serum biomarkers that are being used for diagnosis, prognosis and surveillance of testicular cancer. These serum markers include alpha feto protein, human chorionic gonadotrophin and lactase dehydrogenase. AFP is a $70 \mathrm{kD}$ glycoprotein produced by fetal yolk sac, liver and gastrointestinal tract. Increased levels of AFP are typically found in non seminomatous tumors (embryonal carcinoma and yolksac (Figure 1B). AFP levels are typically not elevated in seminomas; however if increased levels of AFP are found in pure seminoma, it must be considered and treated as non seminomatous germ cell tumor. Elevated serum HCG levels are typically present in both seminomas and non seminoma. Increased levels of serum HCG following orchiectomy is an indication of persistent disease, where as recurrence of HCG following chemotherapy-induced complete remission of metastatic disease indicates the presence of relapse.LDH is less specific marker but has an independent prognostic value in men with advanced testicular cancer. It has been reported that LDH reflects the growth rate and tumor burden. Germa-Lluch JR, et al. in study of 1490 cases noted that tumor markers were raised in $21 \%$ of seminomas and $79 \%$ of Non-seminomatous germ cell tumors [30]. 


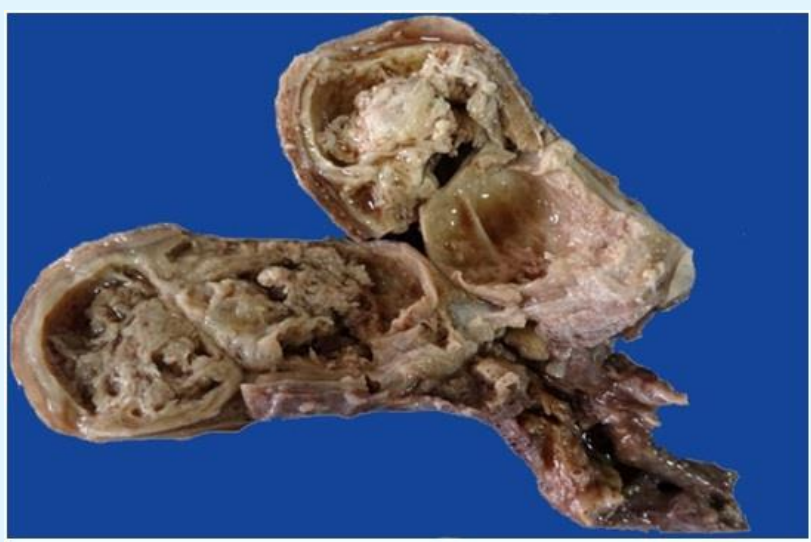

Figure 1B: Gross specimem of testis with Mixed Non Seminomatous germ cell tumor Cut section of growth revealing a cystic cavity (shown by arrow) with adjacent fragile grey white tumor. Testicular parenchyma is reduced to a thin rim at the periphery.

$\mathrm{LDH}$ is raised in $40-60 \%$ of patients with testicular germ cell tumors [31] with limited sensitivity and specificity for seminomatous component, but LDH levels $>2000$ ukat/l are more consistent with bulky disease, and rising levels are an accurate indicator of recurrence. In NSGCT's, LDH is directly related to tumor burden [32].

Tumor marker studies in testicular cancer help in diagnosing and planning the treatment of the tumors, however, the diagnosis of testicular cancer cannot be made based on elevated tumor markers alone, as these markers lack sufficient sensitivity and specificity. Diagnosis instead relies on the histopathologic examination of the orchiectomy specimen.

The degree of tumor marker elevation is directly proportional to tumor burden, and higher serum levels of these tumor markers have been recognized as poor prognostic indicators. According to risk stratification system given by International germ cell cancer collaborative group classification (IGCCCG), seminomas can have good or intermediate prognosis depending upon the degree of tumor marker elevation and presence or absence of non-pulmonary visceral metastasis. Seminomas never have bad prognosis. The degree of tumor marker elevation in patients with NSGCT's has been incorporated with staging criteria to stratify patients into good, intermediate, and poor risk groups according to IGCCCG.

\section{Pathology: Gross Examination}

Seminoma has a characteristic gross appearance. It is usually of moderate size solid homogenous and light yellow and may contain sharply circumscribed zones of necrosis. Areas of cystic change and hemorrhage are not seen [19]. Embryonal carcinoma has grossly more variegated appearance than seminoma. It is mainly solid and gray or white with area of haemorrhage and necrosis. Teratoma is predominantly cystic and multiloculated, foci of cartilage are usually evident the presence of bone is infrequent. Teratocarcinomas are multicystic in areas of teratoma and solid with areas of haemorrhage and necrosis in areas of embryonal carcinoma. Choriocarcinomas are often small with no enlargement of testis. They are usually haemorrhagic and partly necrotic. Pure yolk sac tumor of infancy has a soft consistency and a microcystic appearance on cut section. Leydig cell tumor presents as generally small, sharply delimited solid nodule embedded within the testicle. The brown color it exhibits is one of its most distinguishing gross features. Sertoli cell tumors are well circumscribed white or yellow and firm with focal cystic areas. Testicular lymphomas grossly result in a solid homogenous replacement of testicular parenchyma similar to that of seminoma.

\section{Microscopic Examination}

The vast majorities of testicular tumors are of germ cell origin and, like the totipotent germ cells from which they arise, may differentiate along several pathways. The distinction of seminoma from the non-seminomatous germ cell tumors remains of critical clinical importance. Tumors of non-germinal origin, although relatively uncommon, are frequent diagnostic problems, both from the standpoint of classification and prognosis. The mesothelium that lines the tunica vaginalis testis may give rise to mesotheliomas, as well as provide the basis, through mullerian metaplasia, for the formation of epithelial tumors more typically identified in the ovary [33].

The overwhelming majority of primary testicular tumors are of germ cell origin. The new World Health Organization (WHO) classification 2016 of testicular tumors is given in Table 1 [1]. Some important changes have been made in the WHO classification of testicular tumours in 2016 compared with that adopted in 2004. These revisions were discussed over the course of several months in 2014 through e-mail communications and were finalized in Zurich, Switzerland, in March 2015. The most significant differences between the newly published classification and the prior version concern the germ cell tumours, but other categories are also affected. The comparison of relative incidence of various histological types in different series is a difficult task. The international histological classification published by WHO 
(2004) has been an important landmark in bringing unanimity in the study of testicular tumors all over the world. Small differences in the incidence of germ cell tumors in various studies may be due to different sample sizes. Chitale A and Vadera V have noted 142 germ cell tumors of one histological type out of total 154 germ cell tumors $(92.20 \%)$ [34].
The percentage of seminomas in all testicular tumors in various series ranges from $30.43 \%$ to $53.6 \%$. Fischer C.G. et al in a study of 6594 cases with testicular tumors reported3327 cases of seminoma amounting to $53.6 \%$ of the Germ cell tumors [23] where as Shirish C, et al. noted 7 seminomas which constituted $37.78 \%$ of 45 Germ cell tumors and $47.22 \%$ of 36 Germ cell tumors of one histological type [20].

\section{Germ cell tumours derived from germ cell neoplasia in situ \\ Non invasive germ cell neoplasia \\ Germ cell neoplasia in situ \\ Specific forms of intratubular germ cell neoplasia,}

Tumours of a single histological type (pure forms) Seminoma

Seminoma with syncytiotrophoblastic cells

Nonseminomatous germ cell tumor

Embryonal carcinoma

Yolk sac tumour postpubertal type

Trophoblastic tumours

Choriocarcinoma

Non choriocarcinomatous trophoblastic tumor

Placental site trophoblastic tumour

Epitheloid trophoblastic tumor

Cystic trophoblastic tumor

Teratoma, postpubertal type

Teratoma with somatic type malignancy

Monodermal teratoma

Teratoma with somatic type malignancies

Non seminomatous germ cell tumor of more than one

histological type (mixed forms)

Mixed germ cell tumor

Germ cell tumors of unknown type

Regressed germ cell tumors

Germ cell tumors unrelated to germ cell neoplasia in situ

Spermatocytic tumor

Teratoma prepubertal type

Dermoid cyst

Epidermoid cyst

Well differentiated neuroendocrine tumor (monodermal teratoma)

Mixed teratoma and yolksac tumor, prepubertal type

Yolksac tumor, prepubertal type

Sex cord stromal tumors

Pure tumors

Leydig cell tumour

Malignant Leydig cell tumour
Sertoli cell tumour

Malignant Sertoli cell tumour

Large cell calcifying Sertoli cell tumour

Intratubular large cell hyalinising sertoli cell neoplasia

Granulosa cell tumour

Adult type granulosa cell tumour

Juvenile type granulosa cell tumour

Tumours in the thecoma/fibroma group

Mixed and unclassified sex cord stromal tumors

Mixed Sex cord/stromal tumour:

Unclassified Sex cord-stromal tumours,

Tumours containing both germ cell and sex Cord-stromal elements

Gonadoblastoma

Miscellaneous tumours of the testis

Ovarian epithelial type tumors

Serouscystadenoma

Serous tumor of borderline malignancy

Well differentiated endometrioid carcinoma

Serouscystadenocarcinoma

Mucinouscystadenoma

Mucinous borderline tumor

Mucinous cystadenocarcinoma

Endometroid adenocarcinoma

Clear cell adenocarcinoma

Brenner tumor

Juvenile Xanthogranuloma

Hemangioma

Haematolymphoid tumours

Diffuse large B cell lymphoma

Follicular lymphoma NOS

Extranodal NNK/T cell lymphoma nasaltype Plasmacytoma

Myeloid sarcoma

Rosai dorfman disease

Tumours of collecting ducts and rete testis Adenoma

Adenocarcinoma

Table 1: Classification of testicular tumors (2016). 
Granulomatous inflammation may be associated in $20 \%$ of cases [35]. Invasion of epididymis and spermatic cord is noted in 10-15\% cases [17,36] Extension beyond capsule to scrotal skin, epididymis and spermatic cord is seen in $8 \%$ cases. It has been recognized for several decades that the majority of germ cell tumours arise from progression of an intratubular malignant germ cell that has the morphologic and immunohistochemical features of a seminoma cell. Such cells were usually designated as either carcinoma in situ (CIS) or intratubular germ cell neoplasia, unclassified (IGCNU), with those terms receiving preferential use in Europe and North America, respectively. The main objection to CIS was that the malignant germ cells to which it referred were not epithelial (a similar objection applied to testicular intraepithelial neoplasia), whereas IGCNU, be-cause it contained the word unclassified, was felt to falsely convey an element of doubt concerning the nature and clinical behaviour of the lesion. Germ cell neoplasia in situ (GCNIS) was proposed as an alternative term, retaining the in situ nomenclature that correctly conveys the fact that the lesion is a well-established precursor to an invasive germ cell tumour and simultaneously does away with both the mis leading epithelial-based nomenclature and the use of the word unclassified.

GCNIS is now the WHO-recommended term for this precursor lesion, and other forms of intratubular neoplasia should be referred to by their differentiated phenotype with the prefix intratubular A new emphasis in WHO 2016 is the distinction of GCNIS from maturationdelayed germ cells, which are a relatively common feature in the gonads of patients with disorders of sex development. It is believed that maturation-delayed germ cells likely give rise to GCNIS, but they should be distinguished from the latter because progression is not invariable. Unfortunately, both lesions express the usual markers used for the identification of GCNIS (eg, OCT3/4, placental alkaline phosphatase, AP-2g). The differentiation of the two relies on the more diffuse distribution and central tubular location of maturationdelayed germ cells (as well as the lack of expression of KIT ligand (stem cell factor) in the associated seminiferous tubules, which contrast with the findings in GCNIS.

The prior version of the WHO classification was purely morphologically based and divided the germ cell tumours into those of single or more than one histologic type. In this process, many dissimilar tumours came to be placed under similar diagnostic terms. The new approach recognizes that there are significantly different pathogeneses for testicular germ cell tumours, despite only subtle or even no perceptible morphological differences the germ cell tumours are now broadly separated into two fundamentally different groups: those derived from GCNIS and those unrelated to GCNIS. It is apparent, however, that the latter group is heterogeneous. In contrast, the former group shows a number of basic similarities despite varied morphologies and, to some extent, behaviours. The GCNIS-derived tumours have comparable epidemiologic associations and usually occur in a background of perturbed testicular development that has recognizable morphologic features: impaired spermatogenesis, tubular shrinkage, peritubular Sclerosis, immature Sertoli cells, interstitial widening, hyalinized tubules and microlithiasis. They share the finding of amplification of genetic material from the short arm of chromosome 12 , often in the form of isochromosome $12 p$, and represent progression from GCNIS, often through at least a transient stage of seminoma that may be intratubular. Earlier seminomas were classified into three main types Histologic characteristics of anaplastic seminoma include increased mitotic activity ( $\geq 6$ mitoses per high-power field), nuclear pleomorphism, and cellular anaplasia [37]. Differentiating anaplastic from classic seminoma is worthwhile, as up to $30 \%$ of patients who die from seminoma have anaplastic morphology. Features suggesting greater aggressiveness of this subtype include increased mitotic activity, higher rate of local invasion, and increased rate of metastatic spread. However, when compared stage to stage, treatment outcomes for patients with classic versus anaplastic seminoma have been similar, suggesting that anaplasticseminoma may have a greater metastatic potential and patients with this subtype may present at an advanced stage [37].

Spermatocytic tumor (spermatocytic seminoma) accounts for $2 \%$ to $12 \%$ of all seminomas, and approximately half occur in men older than 50 years. Cells usually vary in size and have deeply pigmented cytoplasm and rounded nuclei containing characteristic filamentous chromatin (Figure 2A). These tumors tend to have an extremely low metastatic potential and a favorable prognosis [34]. The frequency of spermatocytic seminoma in germ cell tumors described by various authors varies from 1.1-7.4\% [17,19,37,38]. Spermatocytic tumor lacks association with GCNIS and shows a unique amplification at chromosome 9 in contrast to $12 \mathrm{p}$ amplification present in case of seminomas. This is the reason that the term spermatocytic seminoma has been replaced by spermatocytic tumor and is placed in separate category of germ cell tumors unrelated to germ cell neoplasia in situ. 


\section{Clinical Pathology \& Research Journal}

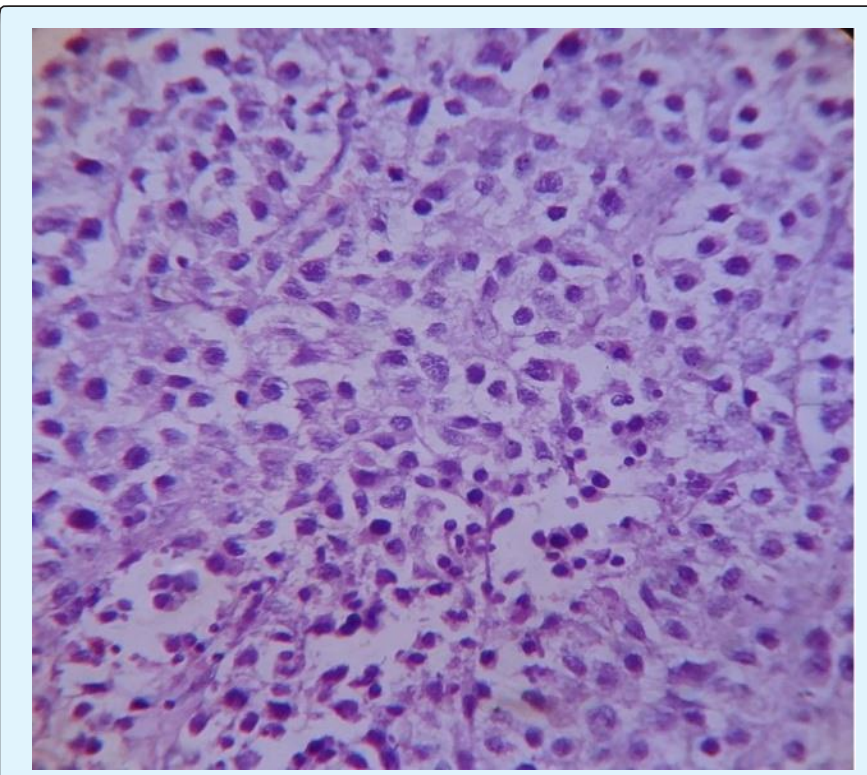

Figure 2A: Photomicrograph showing round cells with a distinct cell membrane. Cells are uniform with abundant cytoplasm showing clearing. Nuclei are centrally located with clumped chromatin (Haematoxylin \& Eosin, 400x) Classical Seminoma.

The frequency of teratoma reported by various authors range from 1.2 to $25.13 \%$ [17,34]. Teratomas in children and the dermoid cyst are benign. Agarwal PK and Palmer JS reported $48 \%$ cases of teratoma in pre-pubertal males [39]. The well differentiated, mature tissue types consist of keratinizing and non keratinizing squamous epithelium, neural and glandular tissues. Organoid structures are not uncommon, particularly in children such as skin, respiratory, gastrointestinal and genitourinary tract. Thyroid tissue has rarely been observed of the mesodermal components, muscular tissue is the most common. Virtually any other tissue type can be seen. Fetal type tissue may also consist of ectodermal, endodermal and/ormesenchymal tissues (Figure 2B). They can have anorganoid arrangement resembling primitiverenal or pulmonary tissues. The prepubertal type teratom as have also been categorized as Germ cell tumor unrelated to germ cell neoplasia in situ in WHO 2016 Classification as they lack GCNIS and 12p amplification and do not occur in malformed testis.

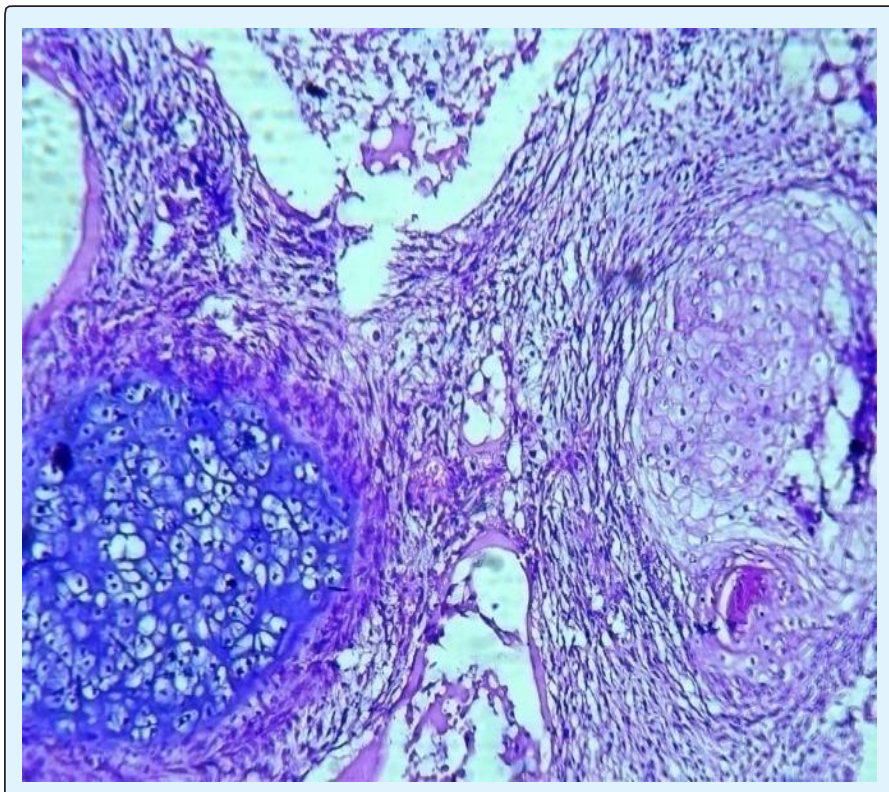

Figure 2B: Photomicrograph showing mature component of Teratoma. Cartilage with mature mesenchymal tissue is seen (Haematoxylin \& Eosin, 400x).

Same was found true for yolk sac tumor (prepubertal type) The frequencies of yolk-sac tumor recorded in various studies range from $2.4-20 \%$ [17,19,39]. Several different patterns are usually admixed, and may be present in equal amounts, although not in frequently one pattern may predominate. Tumors composed entirely of a single histologic pattern are rare. Embryonal carcinoma is a tumour composed of undifferentiated cells of epithelial appearance with abundant clear to granular cytoplasm and a variety of growth patterns. The growth pattern varies from solid and syncytial to papillary with or without stromal fibro vascular cores, forming clefts or gland-like structures. .The frequency of embryonal carcinoma in various series ranged from 4.2 to $37 \%$ of germ cell tumors. This is due to different interpretation of this term by different workers and inclusion of yolk-sac tumors in adult embryonal carcinomas. Cases of embryonal carcinoma show areas of hemorrhage and necrosis and infiltration into capsule. The tumor cells are undifferentiated and of epithelial appearance, cytoplasm is abundant, usually finely granular but may also be clear. Cell borders are indistinct and the cells show overlapping [36]. 
The percentage in various series shows wide range. Shirish C, et al. recorded 20\% of cases with Germ cell tumors of more than one histological type [22]. Figures as high as $62 \%$ are described by some authors [36]. Embryonal carcinoma and seminoma was the commonest combination reported by Shirish C, et al. (33.33\%) while the combination of teratoma and embryonal carcinoma (teratocarcinoma) was the commonest combination noted by Mostofi FK, et al. [36]. Early metastasis with poor prognosis is seen in tumors with embryonal carcinoma element. Presence of seminoma does not alter the prognosis, while as the presence of choriocarcinoma element suggests grave prognosis. The average age of patients of germ cell tumor of more than one histologic type is 33.77 years with age range from 21 years to 43 years [17].

Among the unusual tumors of testis Leydig cell tumors comprise between $1 \%$ and $3 \%$ of all testicular tumors. about $3 \%$ are bilateral either synchronously or metachronously. Microscopically the tumor cells have well defined outlines, deeply acidophilic but occasionally clear cytoplasm and a round or oval nucleus. Some of the cells have plasmacytoid appearance. The frequency of sex-cord stromal tumors in various series is reported as 3 to $4 \%$.

Percentage of lymphoma in various series ranged from 1 to 8\% [34]. The gross and microscopic features of lymphoma are same in many studies. On microscopy in various studies DLBCL is the most common primary testicular lymphoma and constitutes about $27-75 \%$ of primary testicular lymphomas [40-43].

Miscellaneous group includes very rare tumors which can occur in testis. Extramedullary involvement is frequent in cases of acute lymphoblastic leukemia with particular predilection for CNS, lymphnodes and testis in male patients. 25 out of $35(65 \%)$ ALL cases in boys presented with leukaemic infiltration into the testis in a survey done by $\mathrm{H}$ Reid et al in children hospital Royal Manchester. This survey supported the view that testicular infiltration is an indication of wide spread disease.

Metastasis to testes is rare except for lymphoma and leukemia. These lesions are typically incidental findings at autopsy in about $0.02 \%$ to $0.06 \%$ of men with malignant tumors. The ratio may be as high as $2.5 \%$ if the testis is thinly sliced. Most common primary malignancies metastasizing to testes arise from prostate, followed by the lung, gastrointestinal tract, melanoma and kidneys [44].

\section{Immuno Histochemical Markers for Testicular Tumors}

OCT 4 is the stem cell marker very sensitive and specific for seminoma and embryonal carcinoma. SOX2 is also a stem cell marker expressed in embryonal carcinoma SALL4 is again a stem cell marker and a pan germ cell tumor marker, primary utility being to identify Yolk sac tumor which are negative for OCT4 (Figure 2C).

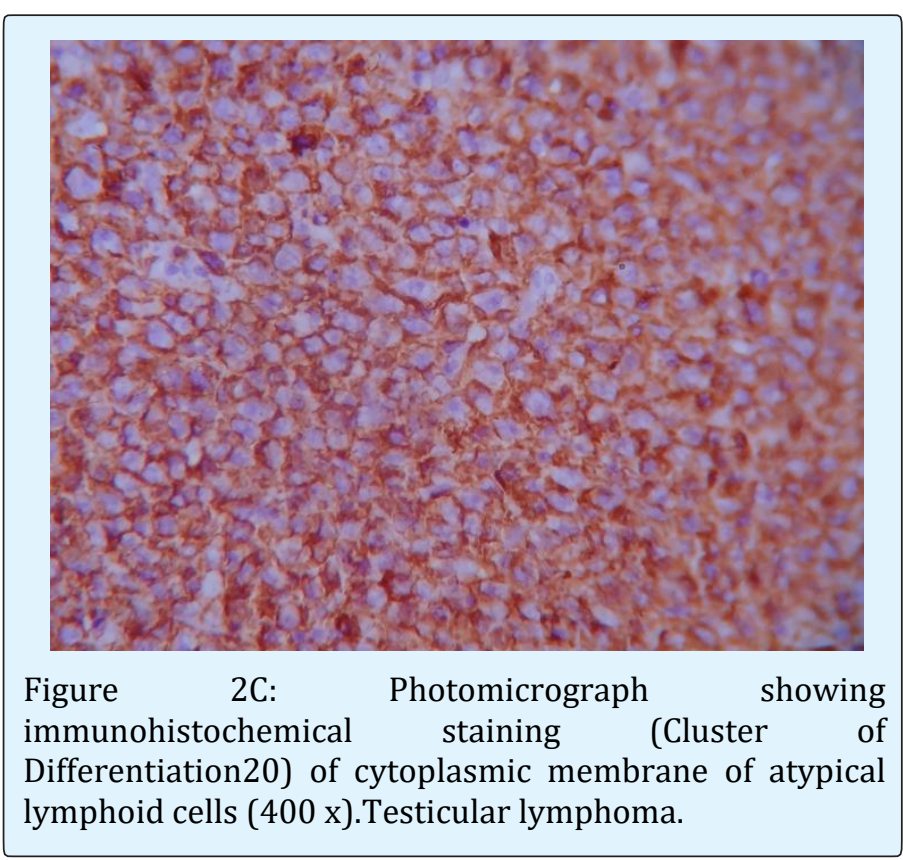

D2-40 Podoplanin is mesothelial and lymphatic marker which also identifies seminoma and embryonal carcinoma. Epithelial markers like cytokeratins and EMA can also be used. All non seminomatous germ cell tumors are cytokeratin positive but EMA negative while as seminoma is negative for all epithelial markers $[45,46]$.

\section{Staging}

Once a diagnosis of testicular cancer is made (almost always after radical orchiectomy: the removal of the testis and spermatic cord), treatment decisions are primarily based on clinical staging, which usually consists of computed tomography (CT) imaging of the chest, abdomen, and pelvis. Patterns of metastatic spread in testicular cancer are very predictable, and the reproducibility of expected "landing sites" aids tremendously with staging and treatment decision making. In the simplest form, staging is divided into localized (testis only), retroperitoneal (minimal or bulky), and extralymphatic/extra-retroperitoneal metastatic disease. The staging data are combined with levels of serologic tumor markers to guide management. The prevalence of several different staging schemes, as well as 
the complexity of the chosen tumor, nodes and metastases (TNM) system, has confounded data acquisition and outcome reporting. The currently recommended staging system for testicular tumors is the
American Joint Committee on Cancer (AJCC) tumor- node - metastasis (TNM) classification as given in (Table 2) [33].

\begin{tabular}{|c|c|c|c|c|c|}
\hline \multicolumn{5}{|c|}{ TNM System } & Stage Grouping \\
\hline \multicolumn{5}{|c|}{ TX: Unknown status of testis } & Stage 0: Tis, N0, M0, S0 \\
\hline \multicolumn{5}{|c|}{ T0: No apparent primary (includes scars) } & Stage IA: T1, N0, M0, S0 \\
\hline \multicolumn{5}{|c|}{ Tis: Intratubular tumor, no invasion } & Stage IB: T2-T4, N0, M0, S0 \\
\hline \multirow{2}{*}{\multicolumn{5}{|c|}{$\begin{array}{l}\text { T1: Testis and epididymis only; no vascular invasion or penetration of } \\
\text { tunica albuginea }\end{array}$}} & Stage IS: any T, N0, M0, S1-S3 \\
\hline & & & & & Stage IIA: any T, N1, M0, S0-S1 \\
\hline \multirow{2}{*}{\multicolumn{5}{|c|}{$\begin{array}{l}\text { T2: Testis and epididymis with vascular invasion or through tunica } \\
\text { albuginea to involve tunica vaginalis }\end{array}$}} & Stage IIB: any T, N2, M0, S0-S1 \\
\hline & & & & & Stage IIC: any T, N3, M0, S0-S1 \\
\hline \multicolumn{5}{|c|}{ T3: Spermatic cord } & Stage IIIA: any T, any N, M1a, S0-S1 \\
\hline \multicolumn{5}{|c|}{ T4: Scrotum } & Stage IIIB: any T, any N, M0-M1a, S2 \\
\hline \multicolumn{5}{|c|}{ NX: Unknown nodal status } & Stage IIIC: any T, any N, M0-M1a, S3 any T, \\
\hline \multicolumn{5}{|c|}{ N0: No regional node involvement } & any $\mathrm{N}, \mathrm{M} 1 \mathrm{~b}$, any S \\
\hline \multicolumn{6}{|c|}{ N1: Node mass or single nodes $\leq 2 \mathrm{~cm} ; \leq 5$ nodes involved; no node $>2 \mathrm{~cm}$} \\
\hline \multicolumn{6}{|c|}{$\begin{array}{c}\text { N2: Node mass }>2 \text { but }<5 \mathrm{~cm} \text {; or }>5 \text { nodes involved; none }>5 \mathrm{~cm} \text {; or } \\
\text { extranodal tumor }\end{array}$} \\
\hline \multicolumn{6}{|c|}{$\mathrm{N} 3:$ Node mass $>5 \mathrm{~cm}$} \\
\hline \multicolumn{6}{|c|}{ MX: Unknown status of distant metastases } \\
\hline \multicolumn{6}{|c|}{ M0: No distant metastases } \\
\hline \multicolumn{6}{|c|}{ M1a: Non regional nodal or lung metastases } \\
\hline \multicolumn{6}{|c|}{ M1b: Distant metastasis other than non regional nodal or lung } \\
\hline \multicolumn{6}{|c|}{ SX: No marker studies available } \\
\hline \multicolumn{6}{|c|}{ S0: All marker studies normal } \\
\hline & $\mathrm{LDH}^{a}$ & & $\mathrm{hCG}(\mathrm{mIU} / \mathrm{mL})$ & & $\mathrm{AFP}(\mathrm{ng} / \mathrm{mL})$ \\
\hline S1: & $<1.5 \times \mathrm{N}$ & and & $<5000$ & and & $<1000$ \\
\hline S2: & $1.5-10 \times \mathrm{N}$ & or & $5000-50,000$ & or & $1000-10,000$ \\
\hline S3: & $>10 \times \mathrm{N}$ & or & $>50,000$ & or & $>10,000$ \\
\hline & & $\mathrm{H} \mathrm{le}$ & expressed as elev & bove upper & imit of normal $(\mathrm{N})$. \\
\hline
\end{tabular}

Table 2: Classification of tumor- node - metastasis (TNM).

\section{References}

1. Moch H, Humphrey PA, Ulbright TM, Reuter V (2016) WHO Classification of Tumours of the Urinary System and Male Genital Organs. Lyon, France: International Agency for Research on Cancer.

2. Eble JN, Sauter G, Epstein JI, Sesterhenn IA (2004) World Health Organization Classification of Tumours. Pathology and Genetics of Tumours of the Urinary System and Male Genital Organs. Lyon, IARC Press, France.

3. Moller $H$ and Evans $H$ (2003) Epidemiology of gonadal germ cell cancer in males and females 111(1): 43-48.
4. Jemal A, Siegel R, Ward E, Hao Y, Xu J, et al. (2009) Cancer statistics. CA Cancer J Clin 59(4): 225-249.

5. Carver BS, Sheinfeld J (2005) Germ cell tumors of testis. Ann Surg Oncol 12(11): 871-880.

6. Baade P, Carriere P, Fritschi L (2008) Trends in testicular germ cell cancer incidence in Australia. Cancer Causes Control 19(10): 1043-1049.

7. Tongaonkar H (2010) Testicular cancer: Current management and controversial issues. Indian J Urol 26(1): 63 . 


\section{Clinical Pathology \& Research Journal}

8. McGlynn KA, Cook MB (2009) Etiologic factors in testicular germ cell tumors. Future Oncol 5(9): 13891402.

9. Dieckmann KP, Pichlmeier U (2004) Clinical epidemiology of testicular germ cell tumors. World J Urol 22(1): 2-14.

10. Richiardi L, Akre O, Bellocco R, Ekbom A (2002) Perinatal determinants of germ-cell testicular cancer in relation to histological subtypes. Br J Cancer 87(5): 545-550.

11. Lee PA, Mark FB, Coughlin MT (1998) Correlations among hormone levels, sperm parameters and paternity in formerly unilaterally cryptorchid men. J Urol 160: 1155-1157.

12. Petridou E, Roukas KI, Dessypris N, Aravantinos G, Bafaloukas D, et al. (1997) Baldness and other correlates of sex hormones in relation to testicular cancer. Int J Cancer 71(6): 982-985.

13. Van den Eeden SK, Weiss NS, Strader CH, Daling JR (1991) Occupation and the occurrence of testicular cancer. Am J Int Med 19(3): 327-337.

14. Frisch M, Biggar RJ, Engels EA, Goedert JJ (2001) Association of cancer with AIDS-related immunosuppression in adults. JAMA 285(13): 17361745 .

15. Hemminki K, Li X (2004) Familial risk in testicular cancer as a clue to a heritable and environmental aetiology. Br J Cancer 90(9): 1765-1770.

16. Tan GH, Azrif M, Shamsul AS, Praveen S, Goh EH, et al. (2011) Clinico-pathological features and survival of testicular tumors in a Southeast Asian university hospital: a ten-year review. Asian Pac J Cancer 12(10): 2727-2730.

17. Shirish Chandanwale, Ramanpreet Chawla, Archana Buch, Arpana Dharwadkar, Charusheela Gore, et al. (2012) Pathology of testicular tumors-Review of 50 cases. Int J Pharm Biomed Sci 3(4): 167-176.

18. Mahalik SK, Prasad A, Bhalla S, Kulshreshtha R (2011) Transilluminating testicular mass. Indian J Paediatr Oncol 32(1): 46-48.

19. Rosai J (2009) Male reproductive system. In: Rosai and Ackerman's Surgical pathology. 9th (Edn.), Juan Rosai (Ed.), St. Louis Mosby, USA, pp: 1412-1533.
20. Fischer CG, Waechter W, Kraus S, Perez EF, Weidner W, et al. (1998) Urologic tumors in the Federal Republic of Germany: data on 56,013 cases from hospital cancer registries. Cancer 82(4): 775-783.

21. Albrecht W, Santis MD, Dossenbach GA (2004) Testicular tumor markers: cornerstones in the management of malignant germ cell tumors. J Lab Med 28(2): 109-115.

22. Salako AA, Onakpoya UU, Osasan SA, Omoniyi-Esan GO (2010) Testicular and para-testicular tumors in south western Nigeria. Afr Health Sci 10(1): 14-17.

23. Fergusson L, Alexander AI (2013) Testicular cancer and Cryptorchidism. Front Endocrinol, 4(32): 1-9.

24. Labarthe P, Khedis M, Chevreau C, Mazerolles C, Thoulouzan M, et al. (2008) Management of pure teratoma of the testis in adult, results of a multicenter study over 15 years. Prog Urol 18(13): 1075-1081.

25. Soh E, Berman LH, Grant JW, Bullock N, Williams MV (2008) Ultrasound-guided core-needle biopsy of the testis for focal indeterminate intratesticular lesions. Eur Radiol 18(12): 2990-2996.

26. Mc Donald MW, Reed AB, Tran PT, Evans LA (2012) Testicular tumour ultrasound characteristics and association with histopathology. Urol Int 89(2): 196202.

27. Kravets FG, Cohen HL, Sheynkin Y, Troy Sukkarieh (2006) Intraoperative sonographically guided needle localization of nonpalpable testicular tumors. Am J Roentgenol 186(1): 141-143.

28. Carmignani L, Morabito A, Gadda F, Bozzini G, Rocco F, et al. (2005) Prognostic parameters in adult impalpable ultrasonographic lesions of the testicle. J Urol 174(3): 1035-1038.

29. Fiona Hughes Cassidy, Kevin M Ishioka, Colm J McMahon, Pauline Chu, Kyoko Sakamoto, et al. (2010) MR Imaging of Scrotal Tumors and Pseudotumors Radiographics. The Journal of Continuing Medical Education in Radiology 30(3): 665-683.

30. Germa-Lluch JR, Garcia del Muro X, Maroto P, PazAres L, Arranz JA, et al. (2002) Clinical pattern and therapeutic results achieved in 1490 patients with germ-cell tumours of the testis: the experience of the Spanish Germ-Cell Cancer Group (GG). Eur Urol 2(6): 553-562. 
31. Gilligan TD, Seidenfeld J, Basch EM, Einhorn LH, Fancher T, et al. (2010) American Society of Clinical Oncology Clinical Practice Guideline on Uses of Serum Tumor Markers in Adult Males With Germ Cell Tumors. J Clin Oncol 28(20): 3388-3404.

32. Milose JC, Filson CP, Weizer AZ, Hafez KS, Montgomery JS (2012) Role of biochemical markers in testicular cancer diagnosis staging and surveillance. Open Access J Urol 4: 1-8.

33. Ulbright TM, Berney DM (2010) Testicular and Paratesticular tumors. In: Mills, SE, (Eds.), Sternberg's Diagnostic surgical pathology, 5th (Edn.), Wolters Kluwer, Lippincott Williams and Wilkins, Philadelphia 2: 1944-2006.

34. Chitale A and Vadera V (1992) In: Chitale A and Vadera V (Eds.), Pathology of urinary and male genital systems, B.I. Churchill Livingstone Pvt. Ltd, New Delhi pp: 195-225.

35. Sesterhenn, Isabell A, Davis CJ (2004) Pathology of germ cell tumors of testis. Cancer Control 11(6): 374387.

36. Mostofi FK, David CJ (1985) Male reproductive system and prostate. In: Kissane JM (Ed.), Anderson's Pathology, 8th (Edn.), St. Louis, Torento, Princeton, C V Mosby, 1: 798-824.

37. Fallon B, Joudi FN (2006) Seminoma. HP Urology Board Review Manual 13(1): 1-12.

38. Carrière P, Baade P, Fritschi L (2007) Population based incidence and age distribution of spermatocytic seminoma. J Urol 178(1): 125-128.
39. Agarwal PK, Palmar JS (2006) Testicular and paratesticular neoplasms in prepubertal males. J Urol 176(3): 875-881.

40. Gundrum JD, Mathiason MA, Moore DB, Go RS (2009) Primary testicular diffuse large B cell lymphoma: A population based study on incidence, natural history and survival comparison with primary nodal counterpart before and after the introduction of rituximab. J Clinic Oncol 27(31): 5227-5232.

41. Wang C, Wang H, Wang Q, Shi B (2013) Primary testicular lymphoma; Experience with 13 cases and literature review. Int J Hematol 97(2): 240-245.

42. Miedler JD, Maclennan GT (2007) Primary testicular lymphoma. J Urol 178(6): 2645-2645.

43. Gupta D, Sharma A, Raina V, Bakshi S, Mohali BK (2009) Primary testicular Non Hodgkin Lymphoma; A single institution experience from India ,Indian J Cancer 46(1): 46-49.

44. Lieng Yi Lu, Junne Yih Kuo, Alex TL Lin, Yen Hwa Chang, Kuang Kuo Chen, et al. (2000) Metastatic Tumors Involving the Testes. J Urol ROC 11: 12-17.

45. Dabbs DJ (2010) Diagnostic immunohistochemistry: theranostic and genomic applications. 3rd (Edn.), New York Saunders.

46. Hammerich KH, Ayala GE, Wheeler TM (2008) Application of immunohistochemistry to the genitourinary system (prostrate, urinary bladder, testis and kidney). Arch Pathol Lab Med 132: 432440. 